\title{
So viele Fragen rund um die Ernährung - gibt es Antworten?
}

$\mathrm{D}$ ie Ernährung ist ein täglicher Bestandteil unseres Lebens. Bewusst oder auch unbewusst treffen wir unsere Entscheidung darüber, was wir essen und wie viel wir wovon essen. In der medizinischen Praxis beraten wir unsere Patienten - ambulant mehr als stationär - über die wünschenswerte Ernährung. Dabei stehen die Grundlagen unserer Beratung häufig nur auf wackeligen Beinen. Dies beginnt schon bei der persönlichen Sachkunde: Die Ernährungslehre ist kein umschriebener Teil des Medizinstudiums, bestenfalls wird das Thema im Rahmen der einzelnen Organerkrankungen abgehandelt. Aber auch wenn Ernährungsvorlesungen angeboten werden, stoßen sie in der Regel nur auf eine geringe bis mäßige Resonanz. Und die Ausbildung zum „Ernährungsfachmann“ ist teuer und macht sich meist nicht bezahlt. Kollegen, die sich zum Beispiel auf die Betreuung von Adipositasgruppen spezialisiert haben, können ein Lied davon singen.

Denn bei dem Krankheitsbild der Fettsucht kommt das größte Problem der Ernährungstherapie am augenfälligsten zur Geltung - nämlich die mangelnde Compliance der Patienten. Außerdem ist es außerordentlich schwierig, im Bereich der Ernährung Studien zu planen und zu realisieren, die - wie es heute verlangt wird - evidenzbasiert ausgerichtet sind. Doppelblindstudien sind per se unmöglich, da es natürlich keine PlazeboErnährung geben kann. In vielen Fällen sind die Analysen daher epidemiologisch angelegt und werden zudem zum Teil auch retrospektiv ausgewertet.

Eine positive Ausnahme sind hier die Studien zur Wirkung von Omega-3-Fettsäuren: Ausgehend von epidemiologischen Vergleichen von Fisch oder Fleisch essenden Bevölkerungsgruppen eines Landes wurden in gut geplanten Untersuchungen, zum Beispiel in DART („diet and reinfarction trial“) und insbesondere in der GISSI-Studie (Studie des „Gruppo Italiano per lo Studio della Sopravvivenza nell'Infarto Micardico), die positive Wirkung der Bestandteile des Fischöls herausgestellt.

Andere Ernährungsempfehlungen mussten im Laufe der Zeit modifiziert werden. So haben wir den Einfluss des Nahrungscholesterins sicher lange überschätzt, dagegen hat die Empfehlung, den Konsum tierischer Fette zu reduzieren, nach wie vor einen festen Platz in der Prophylaxe von kardiovaskulären Erkrankungen. Und vor kurzem wurde erstmals eine so grundsätzliche Frage wie die, ob bei der koronaren Herzkrankheit eine Ernährung mit wenig oder viel Kohlenhydraten nützlich ist (AtkinsDiät), wissenschaftlich analysiert. Noch sind jedoch viele Aspekte der Ernährung unklar oder umstritten: Jahrelang diskutiert haben wir beispielsweise die Kochsalzrestriktion bei Hochdruckpatienten. Auch die Haltung zu der Frage, ob Ballaststoffe eine präventive Wirkung auf die Entwicklung eines Kolonkarzinoms haben, hat sich im Laufe der Jahre unterschiedlich entwickelt. Erschwerend kommt hinzu, dass bestimmte Ernährungsformen mit geradezu weltanschaulicher Inbrunst verteidigt werden.

All diesen Schwierigkeiten sollte man sich bewusst sein, wenn man sich mit Ernährung beschäftigt. Es ist unerlässlich, sich selbst ein gewisses Grundwissen anzueignen, um die Patienten gut zu beraten. Entwickelt man durch die Beschäftigung mit dieser Materie noch eine gewisse Begeisterung, ist dies hilfreich. Denn ein Arzt kann einen Patienten nur dann überzeugen, wenn er sich seiner Sache selbst sicher ist - und dafür haben unsere Patienten ein gutes Gespür!

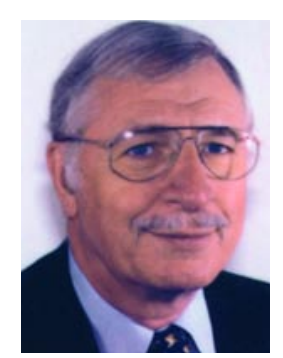

Prof. Dr. A. Weizel, Mannheim

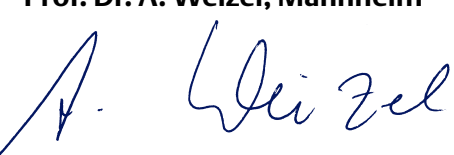

